\title{
CT Perfusion of the Neck: Internal Carotid Artery versus External Carotid Artery as the Reference Artery
}

\begin{abstract}
A.C. Miracle
A. Rezaei

D. Gandhi

S.K. Mukherji

SUMMARY: Arterial selection for reference time-enhancement curve generation in deconvolutionbased perfusion CT (PCT) studies of the head and neck is underevaluated. This study of 11 patients with confirmed head and neck squamous cell carcinoma demonstrates significant correlation (range, $r=0.85-0.95$ ) between perfusion parameter values derived with internal carotid artery (ICA) as compared to an external carotid artery reference, supporting the use of the ICA as arterial reference in PCT studies of the neck.
\end{abstract}

$\mathbf{P}$ erfusion CT (PCT) is a rapid noninvasive imaging technique conceived originally to assess qualitatively and quantitatively regional cerebral blood flow ( $\mathrm{rCBF}$ ), cerebral blood volume (CBV), mean transit time (MTT), and later capillary permeability surface-area product (PS) within regions of interest (ROIs) in the brain. ${ }^{1-4}$ Since its inception, the PCT technique has been adopted for many physiologic imaging applications, including diagnosis and management of head and neck squamous cell carcinomas (HNSCCA). In cerebral PCT, $\mathrm{rCBF}(\mathrm{mL} / 100 \mathrm{~g} / \mathrm{min})$ differs from large-vessel blood flow $(\mathrm{mL} / \mathrm{min})$ because it describes the fractional blood flow (BF) through a mass of tissue (as conducted by microvasculature) rather than flow per unit vascular volume. As such, rCBF, $\mathrm{CBV}$, and MTT are useful measures for describing the perfusion of a parenchymal region of interest. The parameters generated in PCT of the head and neck are defined as fractional tissue $\mathrm{BF}(\mathrm{mL} / 100 \mathrm{~g} / \mathrm{min})$, blood volume (BV, $\mathrm{mL} / 100 \mathrm{~g})$, MTT (seconds), and PS ( $\mathrm{mL} / 100 \mathrm{~g} / \mathrm{min})$. PS, a measure of capillary permeability, was originally used as a marker for blood-brain barrier (BBB) breakdown in ischemic stroke, but PS has also been shown to correspond to the microvascular hyperpermeability seen with neoangiogenesis in brain tumors and extra-axial tumors without a BBB such as HNSCCA. ${ }^{5-7}$ The role of PCT in the diagnosis and management of HNSCCA is continuing to expand and evolve. ${ }^{6-9}$

The deconvolution-based PCT technique is predicated on the central volume principle $(\mathrm{BF}=\mathrm{BV} / \mathrm{MTT})$ and was first described in 1983 by Axel in the context of cerebral perfusion. ${ }^{1}$ An iodinated contrast bolus is monitored as it traverses selected arterial reference, target tissue, and venous ROIs, generating time-enhancement curves proportional to contrast agent concentration. MTT can then be calculated from the arterial and tissue enhancement curves by the mathematic process of deconvolution. Dividing the area under the curve of the tissue pixel by that of the arterial pixel yields BV. The central volume equation is then solved for BF. ${ }^{1-3,10}$

In developmental work on PCT of the brain by Axel, ${ }^{1}$ Lee, and colleagues, the ideal reference artery was postulated to be

Received November 6, 2008; accepted January 4, 2009.

From the Departments of Radiology (A.C.M., A.R., D.G., S.K.M.), Otolaryngology (S.K.M.), and Radiation Oncology (S.K.M.), University of Michigan Health System, University Hospital, 1500 East Medical Center Dr, Ann Arbor, Mich.

Please address correspondence to Aaron C. Miracle, Department of Radiology, University of Michigan Health System, 1500 E. Medical Center Dr, B2B311 UH SPC 5030, Ann Arbor, Ml 48190; e-mail: amiracle@med.umich.edu

DOI 10.3174/ajnr.A1531 a vessel that was nonsupplying, large in caliber, and oriented perpendicular to the viewing plane, minimizing partial volume averaging within the selected ROI. ${ }^{1-4}$ The internal carotid artery (ICA) and other in-plane arteries have since been used to generate adequate reference curves for acute stroke evaluation. ${ }^{3,11}$ Current practices in PCT of the head and neck generally use the ICA, but there is little evidence supporting this practice. ${ }^{6,8}$ The intent of this experiment was to confirm that perfusion parameter values do not significantly differ when the ICA as compared to the external carotid artery (ECA) is used a reference in HNSCCA PCT studies.

\section{Technique}

Fourteen patients with subsequently confirmed HNSCCA who had undergone contrast-enhanced CT and PCT as part of their routine pretreatment clinical evaluation between April and July 2002 were retrospectively evaluated. Our institutional review board approved this investigation. CT scans were obtained on a multidetector scanner (LightSpeed Ultra; GE Healthcare, Milwaukee, Wis). A localizing noncontrast CT was performed through the known primary site, and then 4 adjacent 5 -mm sections at the level of the largest cross-sectional area of the tumor were selected as the area of interest for perfusion measurements. Fifty milliliters of nonionic contrast (iopamidol, Isovue, $300 \mathrm{mg} \mathrm{I} / \mathrm{mL}$; Bracco Diagnostics, Princeton, NJ) was injected at $4 \mathrm{~mL} / \mathrm{s}$. At 5 seconds into the injection, a cine (continuous) acquisition was initiated $(120 \mathrm{kV}, 60 \mathrm{~mA}, 4 \times 5$-mm sections, 1 -second rotation for a duration of 50 seconds). The 1-second images were reformatted at 0.5 -second intervals, and the 5 - $\mathrm{mm}$ sections were reformatted into $10-\mathrm{mm}$ sections.

Perfusion data were postprocessed by using the deconvolution technique with commercially available Perfusion 2 software on an Advantage Windows Workstation (GE Healthcare). A single observer, aware of the primary tumor site, placed a ROI on the ipsilateral ICA, internal jugular vein (IJV), and primary tumor $\left(25-30 \mathrm{~mm}^{2}\right)$, generating contrast enhancement curves (Fig 1). Data were then processed into maps to represent PS, BV, BF, and MTT. Similarly, the same observer then reprocessed the data into maps by placing a ROI on the largest visible branch of the ipsilateral ECA, IJV, and primary tumor (25-30 $\mathrm{mm}^{2}$ ) (Fig 2).

Quantitative measurements of BV, BF, PS, and MTT derived from ICA reference curves were individually compared with similar measurements derived with an ECA reference. Statistical analysis consisted of linear regression and Pearson product moment correlation coefficients. $P<.05$ was considered significant. 


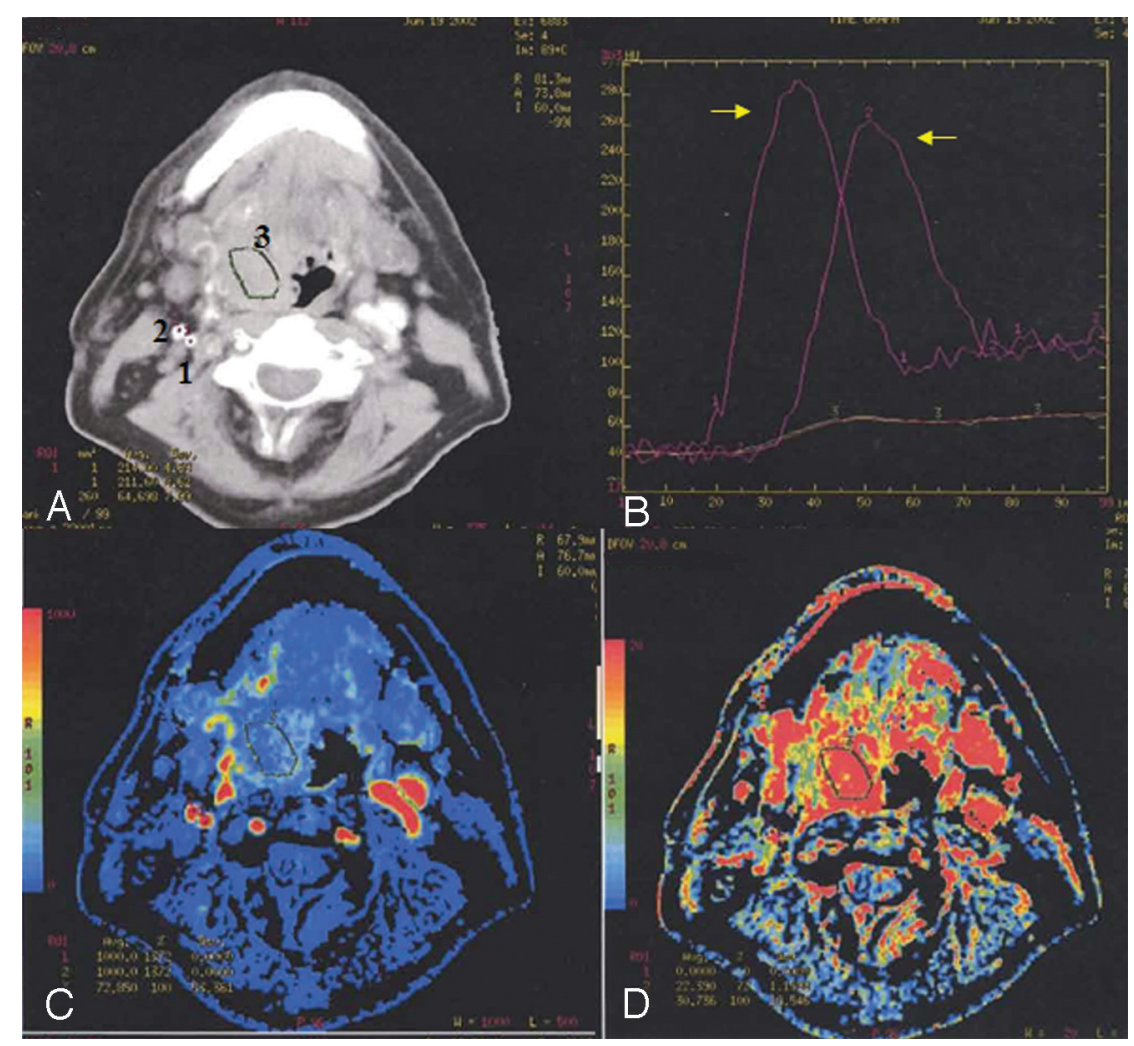

Fig 1. PCT imaging with ICA reference of a patient with right tongue base squamous cell carcinoma. $A$, Axial contrast-enhanced CT scan shows a mass involving the right tongue base Regions of interest have been placed in the ICA (1), the internal jugular vein (2), and the tumor (3). $B$, Time-enhancement curve for the patient shown in $A$. The first peak is arterial enhancement; the second is venous (arrows). The flat curve at the bottom represents tissue region-of-interest (3) enhancement. $C$, Fractional tissue $B F$ map for the patient in $A$. There is increased fractional tissue BF in the region of the mass lesion compared with surrounding tissue. D, PS map for the same patient. The tumor site displays increased capillary permeability compared with surrounding tissue.

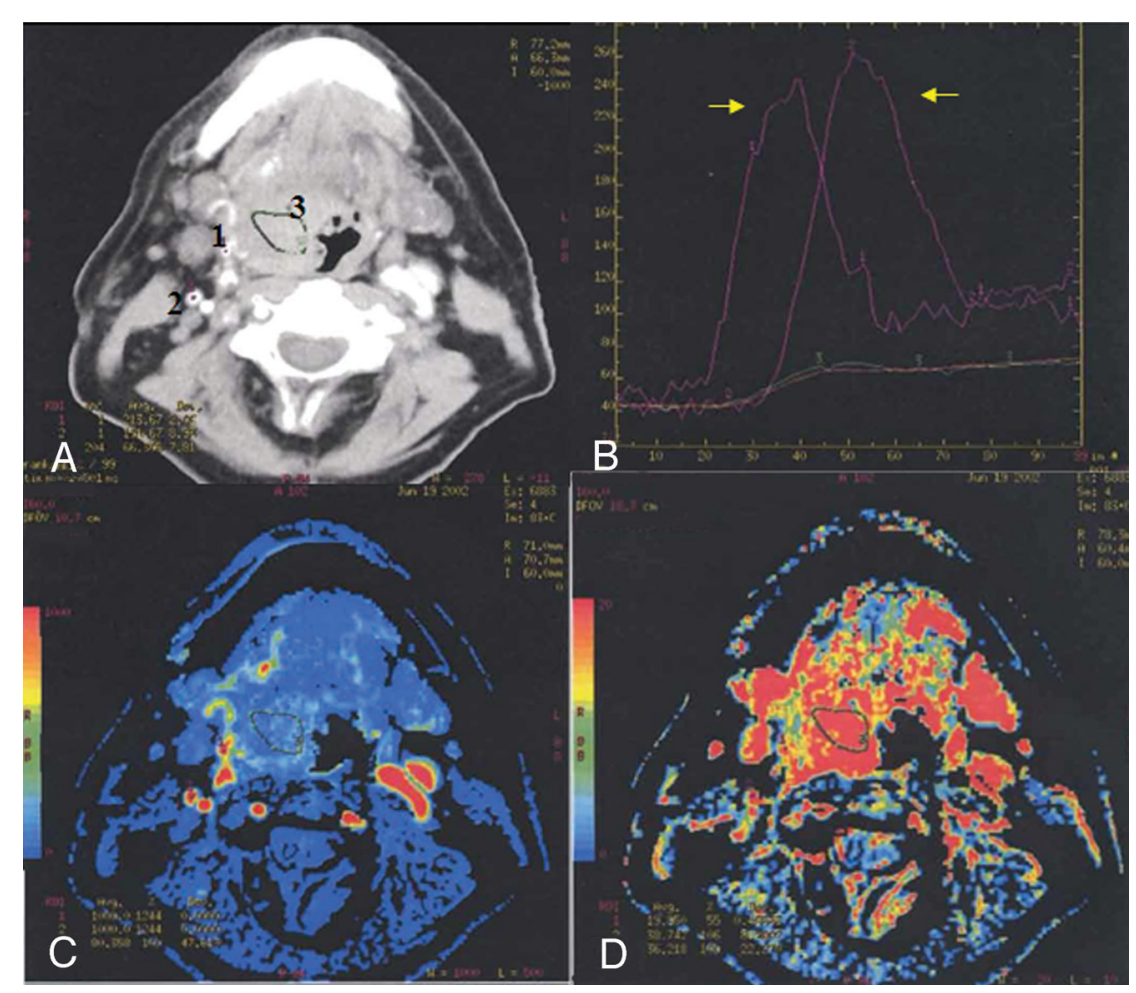

Fig 2. PCT imaging with ECA reference of the same patient depicted in Fig 1. $A$, Regions of interest have been placed in the ECA (1), the internal jugular vein (2), and tumor (3). $B$, Time-enhancement curve for the patient shown in $A$. The first peak is ECA enhancement; the second is venous enhancement (arrows). $C$, The fractional tissue BF map for the patient in $A$ generated with ECA reference is similar to that generated by using the ICA as the reference function. $D$, PS map generated with ECA reference demonstrates increased capillary permeability similar to that generated by using the ICA as reference. 


\begin{tabular}{lccll}
\hline \multicolumn{5}{l}{ Table 1: Summary of patient characteristics } \\
\hline $\begin{array}{l}\text { Patient } \\
\text { No. }\end{array}$ & Age & & & \\
\hline 1 & yr) & Sex & Tumor Location & Stage \\
\hline 3 & 59 & $\mathrm{M}$ & Left tongue base & T4N2cM0 \\
4 & 50 & $\mathrm{~F}$ & Oral tongue & T3NOM0 \\
5 & 49 & $\mathrm{~F}$ & Epiglottis & T2NOM0 \\
8 & 40 & $\mathrm{M}$ & Right floor of mouth & T4N2bM1 \\
9 & 66 & $\mathrm{M}$ & Epiglottis & T3NOM0 \\
10 & 41 & $\mathrm{M}$ & Left buccal area & T3NOM0 \\
11 & 56 & $\mathrm{M}$ & Vallecula & T2N2aM0 \\
12 & 56 & $\mathrm{M}$ & Right tonsil & T4N2bM0 \\
13 & 59 & $\mathrm{M}$ & Right tonsil & T4N2bM0 \\
14 & 72 & $\mathrm{~F}$ & Left tongue base & T4NOM0 \\
\hline
\end{tabular}

\section{Results}

Three of the original 14 patients were excluded on the basis of an inability to reliably identify the ECA. The included study group is depicted in Table 1 and comprised 8 men and 3 women with ages ranging from 40 to 72 years with a mean age of 54.0 years. Primary tumor sites included tonsil (3), tongue (3), buccal area (1), epiglottis (2), vallecula (1), and floor of mouth (1). Tumors were staged according to the tumor-node-
Table 2: Perfusion parameter values derived with ICA and ECA reference* $^{*}$

\begin{tabular}{|c|c|c|c|c|}
\hline Values & ICA & ECA & $\begin{array}{c}\text { Difference } \\
\text { between } \\
\text { Means }\end{array}$ & $P$ Value \\
\hline $\mathrm{BF}(\mathrm{mL} / 100 \mathrm{~g} / \mathrm{min})$ & $89.25 \pm 34.42$ & $90.93 \pm 43.94$ & $-1.67 \pm 15.17$ & .7221 \\
\hline $\mathrm{BV}(\mathrm{mL} / 100 \mathrm{~g})$ & $4.07 \pm 1.46$ & $4.38 \pm 1.78$ & $-0.31 \pm 0.46$ & .0488 \\
\hline MTT (s) & $4.03 \pm 1.36$ & $4.26 \pm 1.19$ & $-0.23 \pm 0.72$ & .3190 \\
\hline $\begin{array}{l}\text { Capillary PS (mL/ } \\
100 \mathrm{~g} / \mathrm{min})\end{array}$ & $27.68 \pm 6.25$ & $26.74 \pm 5.45$ & $0.94 \pm 4.12$ & .4676 \\
\hline
\end{tabular}

Note:- ICA indicates internal carotid artery; ECA, external carotid artery; BF, blood flow: BV, blood volume; MTT, mean transit time; $P S$, permeability surface-area product.

* Data are mean $\pm \mathrm{SD}$.

metastasis system and were predominantly stage III (3) and IV (7) lesions but also included a stage II (1) lesion.

Values for each of BV, BF, MTT, and PS generated using the ipsilateral ICA and ECA for the reference artery were all significantly correlated (Fig 3). Pearson correlation coefficients of $r=0.95$ for BF $(P<.0001), r=0.98$ for BV $(P<$ $.0001), r=0.85(P=.0009)$ for MTT, and $r=0.76(P=.0065)$ for PS were obtained. All correlations were significant with $P<.05$. The mean differences between values derived from ICA compared with ECA reference are included in Table 2.

\section{Blood Volume (BV)}

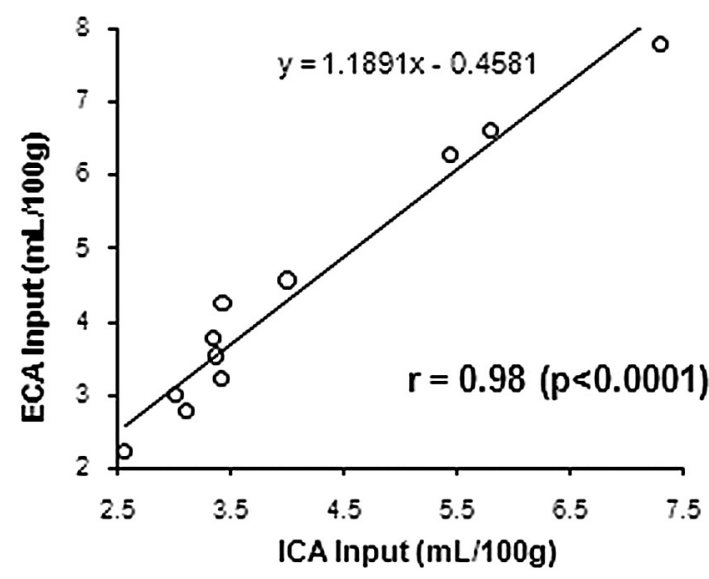

Mean Transit Time (MTT)

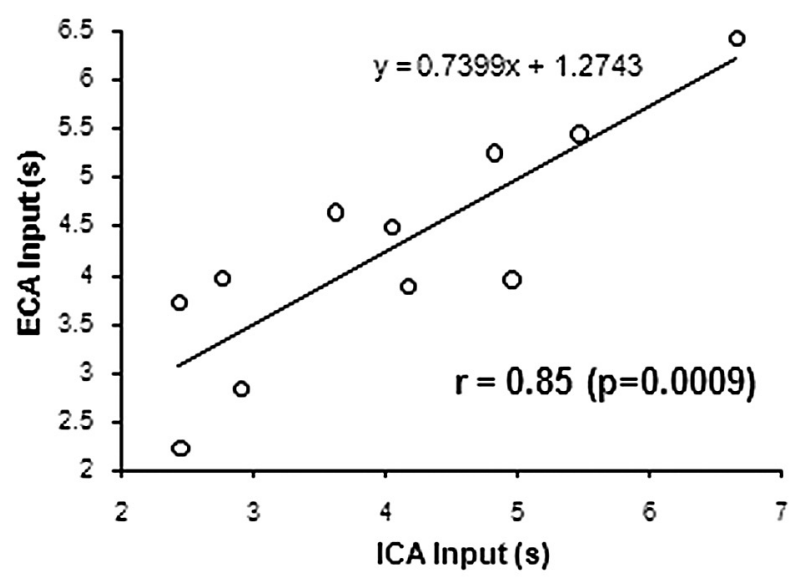

\section{Blood Flow (BF)}

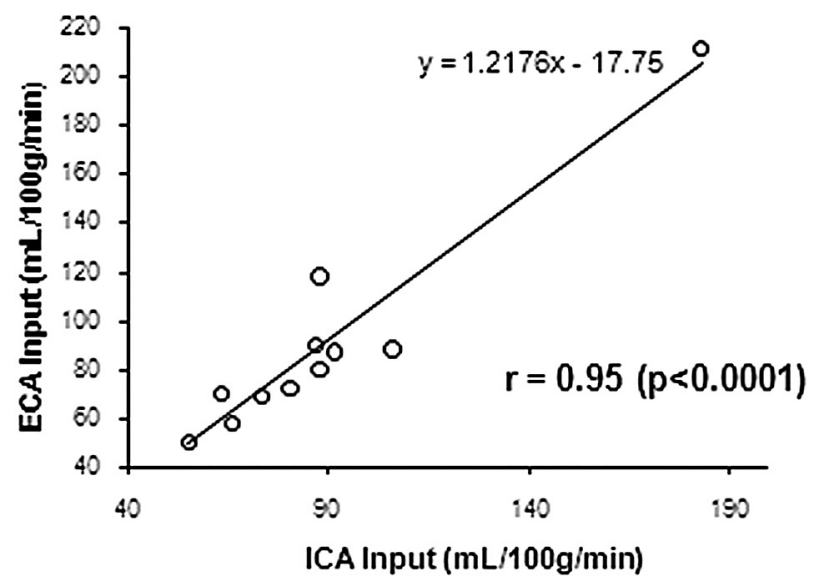

\section{Capillary Permeability Surface Product (CP)}

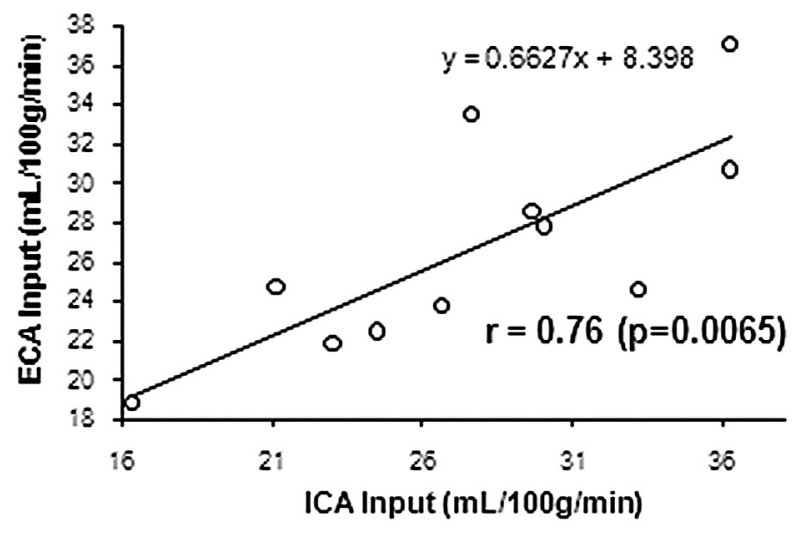

Fig 3. Scatterplots of perfusion parameter measurements derived with an ECA arterial reference compared with the ICA. $r$ values are the Pearson product moment correlation coefficients. All correlations are significant $(P<.05)$ 


\section{Discussion}

Our study specifically focused on a pretreatment population of patients with HNSCCA. The results demonstrated that BV, $\mathrm{BF}$, and MTT derived from ICA reference curves were well correlated with the same measurements from ECA references, with $r$ values ranging from 0.85 to 0.98 . Measurements for PS in our study were the least correlated with $r=0.76$.

Significant correlations between ICA- and ECA-derived parameter values were supported by insignificant differences in the means for all ICA- versus ECA-derived perfusion parameters with the exception of BV (Table 2). The marginally significant difference with $P=.0488$ between $\mathrm{BV}$ mean values could be the result of an insufficient sample size because there is indeed a statistically significant strong correlation $(r=$ 0.98 ) between ICA- and ECA-derived BV values as described above.

In a previous PCT study of benign and malignant head and neck lesions, 4 of which were subsequently determined to be HNSCCA, Rumboldt et $\mathrm{al}^{9}$ found no significant perfusion parameter variance with the use of the ICA compared with ECA reference. The confirmatory evidence presented here supporting the use of ICA reference in HNSCCA PCT studies is important to document as this technique gains greater acceptance for assessing treatment response and differentiating recurrent tumor from posttreatment changes.

Limitations to this study include the relatively small sample size and the exclusion of those patients whose ECAs were not reliably identified in cross-section, conceivably a source of selection bias. If, for example, ill-defined ECA branches were associated with hemodynamically compromised neck vasculature (ie, extensive atherosclerosis with stenoses differentially affecting the ICA compared with the ECA), the correlation between ECA- and ICA-derived parameters could have been overestimated. Also, our study identified the ECA and its branches on noncontrast images. Arterial identification on contrast-enhanced images would be an improvement in future studies.

\section{Conclusions}

This study demonstrated a significant correlation between the PCT measurements of BV, BF, and MTT derived with ICA reference as compared with ECA reference. These results support the use of the ICA as a reference artery in PCT imaging of HNSCCA, though further studies with larger patient populations are needed to confirm these findings.

\section{References}

1. Axel L. Tissue mean transit time from dynamic computed tomography by a simple deconvolution technique. Invest Radiol 1983;18:94-99

2. Cenic A, Nabavi DG, Craen RA, et al. Dynamic CT measurement of cerebral blood flow: a validation study. AJNR Am J Neuroradiol 1999;20:63-73

3. Nabavi DG, Cenic A, Craen RA, et al. CT assessment of cerebral perfusion: experimental validation and initial clinical experience. Radiology 1999;213: 141-49

4. Nabavi DG, Cenic A, Henderson S, et al. Perfusion mapping using computed tomography allows accurate prediction of cerebral infarction in experimental brain ischemia. Stroke 2001;32:175-83

5. Roberts HC, Roberts TPL, Lee T, et al. Dynamic contrast-enhanced computed tomography (CT) for quantitative estimation of microvascular permeability in human brain tumors. Acad Radiol 2002;9(suppl 2):S364-67

6. Bisdas S, Baghi M, Smolarz A, et al. Quantitative measurements of perfusion and permeability of oropharyngeal and oral cavity cancer, recurrent disease, and associated lymph nodes using first-pass contrast-enhanced computed tomography studies. Invest Radiol 2007;42:172-79

7. Bisdas S, Spicer K, Rumboldt Z. Whole-tumor perfusion CT parameters and glucose metabolism measurements in head and neck squamous cell carcinomas: a pilot study using combined positron-emission tomography/CT imaging. AJNR Am J Neuroradiol 2008;29:1376-81. Epub 2008 May 15

8. Gandhi D, Hoeffner EG, Carlos RC, et al. Computed tomography perfusion of squamous cell carcinoma of the upper aerodigestive tract: initial results. J Comput Assist Tomogr 2003;27:687-93

9. Rumboldt Z, Al-Okaili R, Deveikis JP. Perfusion CT for head and neck tumors: pilot study. AJNR Am J Neuroradiol 2005;26:1178-85

10. Wintermark M, Maeder $P$, Thiran JP, et al. Quantitative assessment of regional cerebral blood flows by perfusion CT studies at low injection rates: a critical review of the underlying theoretical models. Eur Radiol 2001;11:1220-30

11. Eastwood JD, Lev MH, Azhari T, et al. CT perfusion scanning with deconvolution analysis: pilot study in patients with acute middle cerebral artery stroke. Radiology 2002;222:227-36 